\title{
Risk factors for postoperative ischemic complications in pediatric moyamoya disease
}

\author{
Xiaofeng Deng ${ }^{1,2,3,4}$, Peicong Ge 1,2,3,4, Rong Wang 1,2,3,4 Dong Zhang ${ }^{1,2,3,4}$, Jizong Zhao 1,2,3,4,5 and \\ Yan Zhang ${ }^{1,2,3,4^{*}}$ (D)
}

\begin{abstract}
Background: Ischemic events are the most common postoperative complication in bypass surgery for moyamoya disease (MMD), but the risk factors for pediatric MMD remain unclear. The goal of the study was to investigate the risk factors for postoperative ischemic complications in pediatric MMD patients.

Methods: We retrospectively reviewed a consecutive series of pediatric MMD cases at Beijing Tiantan Hospital, Capital Medical University from June 2010 through June 2019. Preoperative clinical variables and radiographic findings were recorded, and logistic regression analysis was carried out to identify the risk factors for postoperative ischemic events.

Results: A total of 533 operations in 336 patients were included in this study. Postoperative complications occurred after 51 operations (9.6\%), including 40/447 indirect bypass procedures, 9/70 direct bypass procedures, and 2/16 combined bypass procedures. Postoperative ischemic events were the most common complication and occurred in 30 patients after 31 procedures (8.9\% per patient; 5.8\% per operation), including 26/447 indirect bypass procedures, 4/70 direct bypass procedures, and 1/16 combined bypass procedures, and the incidence of these events did not differ significantly between indirect and non-indirect bypass (5.8\% vs $5.8 \% ; p=0.999)$. Multivariate logistic regression analyses revealed that older age at operation $(\mathrm{OR} 1.129,95 \% \mathrm{Cl} 1.011-1.260, p=0.032)$ and posterior cerebral artery involvement (OR 2.587, 95\% Cl 1.030-6.496, $p=0.043$ ) were significantly associated with postoperative ischemic events.
\end{abstract}

Conclusion: We speculate that older age at operation and posterior cerebral artery involvement are risk factors for postoperative ischemic events in pediatric MMD patients.

Keywords: Moyamoya disease, Pediatric, Risk factors, Ischemic complications

\section{Introduction}

Moyamoya disease (MMD) is an uncommon chronic cerebrovascular occlusive disorder [1]. It is characterized by progressive stenosis or occlusion in the bilateral internal carotid arteries (ICAs) or their main branches with compensatory small arterial collaterals that appear as a "puff of smoke" on angiographic findings [2]. MMD has a low incidence in Western countries, but it is the leading cause

\footnotetext{
*Correspondence: yanzhang135@163.com

1 Department of Neurosurgery, Beijing Tiantan Hospital, Capital Medical University, Beijing 100070, China

Full list of author information is available at the end of the article
}

of stroke in children and adolescents in China, South Korea and Japan [3].

Although controversy remains, revascularization surgery is the primary treatment for MMD; it prevents recurrent stroke and improves the prognosis, especially in the pediatric population [4]. It has been reported that postoperative complications in surgical revascularization for MMD occur in 5.6-20.5\% of patients. Among them, ischemic events are most common (1.5-11.4\%) and may lead to transient neurological deterioration or permanent neurological deficits [4-7]. Therefore, prevention of 
postoperative ischemic complications is very important in ensuring the benefits of surgical revascularization.

Risk factors for postoperative ischemic complications in adult patients with MMD have been well-documented, while the paucity of data on pediatric MMD patients and related evidence is limited [8]. Therefore, we performed this study to investigate the risk factors for postoperative ischemic complications in pediatric MMD.

\section{Materials and methods}

\section{Patient data}

The Ethics Committee of Beijing Tiantan Hospital, Capital Medical University approved this study. All MMD inpatients at Beijing Tiantan Hospital from June 2010 through June 2019 were screened. The patient inclusion criteria were as follows: 1 ) patients diagnosed with MMD, based on digital subtraction angiography (DSA) and/or MR angiography according to the published guidelines of the Research Committee on MMD in Japan [9]; 2) patients who received surgical revascularization; and 3) patients aged less than 18 years old at the time of operation. Patients with a history of brain tumor, cranial irradiation, Down syndrome, neurofibromatosis, meningitis and sickle cell disease were excluded [1].

The patient data collected included age at operation, sex, onset symptoms, admission modified Rankin Scale (mRS) score, past medical history (including hypertension, thyroid disease, hyperlipidemia, smoking, alcohol use, diabetes and family history), surgical modalities, length of hospital stay (LOS), and mRS score at discharge. We categorized the onset symptoms into 3 main types: ischemic-type (infarction and transient ischemic attack (TIA)), hemorrhagic-type (intraventricular hemorrhage, intracerebral hemorrhage, subarachnoid hemorrhage, etc.), and nonspecific-type (headache, epilepsy, asymptomatic, etc.).

\section{Radiologic profiles}

Collateral circulation was evaluated based on the classification criteria reported by Liu et al. [10]. Posterior collateral circulation included 3 parts and was evaluated as follows: (1) on lateral views of vertebrobasilar artery (VA) angiograms, the leptomeningeal collateral networks from the posterior cerebral artery (PCA) territory to the anterior cerebral artery (ACA) territory were given 1 point if blood supply to the cortical border zone between the ACA and PCA territory was present and 2 points if blood supply over the central sulcus via the posterior pericallosal artery we present; (2) on anteroposterior views of VA angiograms, the leptomeningeal collateral networks from the anterior temporal branch of the PCA to the middle cerebral artery (MCA) territory were given 1 point if anastomoses of the anterior temporal branches of the PCA and temporal branches of the MCA were present; (3) also on anteroposterior views of VA angiograms, the leptomeningeal collateral networks from the parietooccipital branch of the PCA to the MCA territory were given 1 point if the retrograde flow of parieto-occipital branch of the PCA extended to the M4 segment of the MCA (superficial vessels only), 2 points if the blood supply extended into the Sylvian fissure and 3 points if the blood supply extended into the occlusion within the M1 or proximal M2 segments.

Anterior collateral circulation was evaluated by Suzuki stage [2], and scores of 6 to 0 were corresponded to Suzuki stages 0 to 6 , respectively.

The sum of the four abovementioned scores was calculated, and the stages of collateral circulation were categorized as follows: Grade I, a score of 0 to 4; Grade II, a score of 5 to 8; and Grade III, a score of 9 to 12 .

\section{Surgical modalities}

Indirect procedures involve placing various connective tissues on the surface of the brain to promote revascularization [4]. In our center, encephaloduroarteriosynangiosis (EDAS) and multiple burr holes $(\mathrm{MBH})$ were performed. For EDAS, the branch of the superficial temporal artery (STA) was placed on the brain surface after being dissected. Intraoperative indocyanine green (ICG) fluorescence angiography was used to ensure the patency of the STA. In terms of MBHs, five to fifteen burr holes were drilled over the hypoperfusion brain areas, and the dura was opened and separated. Direct procedures involved end-to-side anastomosis of the STA to the M4 branch of the MCA. Combined bypass was defined as direct and indirect bypass were both performed on one hemisphere during one operation [11]. The immediate patency of anastomosis in direct and combined bypass was also evaluated by intraoperative ICG fluorescence angiography. Corresponding adjustments must be taken if the arteries were not patent, until patency was ensued by repeated ICG fluorescence angiography.

\section{Postoperative management and complications}

A standardized perioperative management protocol was applied in all cases. All pediatric patients received fluid therapy and blood pressure (BP) monitoring after revascularization. For patients receiving indirect bypass, postoperative BP was controlled greater (no more than $20 \%$ ) than or equal to the baseline BP. For patients with direct and combined bypass, postoperative BP was controlled to be less (no more than 20\%) than or equal to the baseline BP in cases of hyperperfusion syndrome [12]. Patients received a CT scan 4 to $6 \mathrm{~h}$ after the procedure. Repeated CT and/or MRI scans were performed if the patient exhibited postoperative neurological 
deterioration. Postoperative complications were recorded during the hospital stay, including TIA, infarction, cerebral hyperperfusion syndrome, subdural effusion, intracranial infection, seizures, impaired wound healing, and subdural hematoma. Cerebral hyperperfusion syndrome was characterized by a series of neurological deficits, including a focal seizure, reversible deterioration of consciousness level with behavioral and/or aphasia, or intracerebral hemorrhage, along with absence of a definite new infarction on a brain CT scan and/or diffusionweighted MRI. And the diagnosis was further confirmed by a CT perfusion scan [13].

\section{Statistical analysis}

Statistical analysis was performed using SPSS (Windows version 22.0, IBM). Differences between patients with and without postoperative complications were compared by the Student's $t$ test for continuous variables and the chi-square test for categorical variables. The Logistic regression analysis was performed to test which variables were associated with postoperative complications. Clinical variables that achieved a $p$ value $<0.10$ in the univariate analysis were included in the multivariate analysis. A $p$ value $<0.05$ was defined as statistically significant. In addition, the statistical program $\mathrm{R}$ (version 4.0.2; $\mathrm{R}$ core team) was used to create a nomogram.

\section{Results}

\section{Baseline characteristics and the incidence of postoperative} complications

A total of 336 pediatric patients were enrolled in this study. The median time from onset to the first procedure was six months. Among these patients, 144 underwent one revascularization procedure, 187 underwent two operations, and 5 underwent three operations. Overall, a total of 533 procedures were performed, including 447 indirect bypasses, 70 direct bypasses, and 16 combined bypass procedures. The mean age at the time of operation was $9.6 \pm 3.7$ years (Table 1). Based on onset symptoms, the clinical presentation was ischemic-type in 366 cases (68.7\%), hemorrhagic-type in 28 (5.3\%), and nonspecifictype in $43(26.1 \%)$. As shown in Table 1, compared with patients without postoperative complications, patients with postoperative complications tended to be older, and had more cases with PCA involvement and higher collateral circulation grades (all $p<0.05$ ). In addition, patients with postoperative complications had longer hospital stays $(p<0.0001)$ and worse mRS scores at discharge $(p<0.0001)$.

In 533 operations, 55 postoperative complications were observed in 50 patients after 51 revascularization procedures (14.8\% per patient; $9.6 \%$ per operation). Stratified by surgical modality, the number and rate of complications were as follows (Table 2): 40/447 (8.9\%) for indirect bypass, $9 / 70$ (12.9\%) for direct bypass, and $2 / 16(12.5 \%)$ for combined bypass (11/86 for non-indirect bypass, overall), and there was no significant difference between indirect and non-indirect bypass ( 8.9 vs $12.8 \%$; $p=0.267)$.

Postoperative ischemic events (TIAs and infarctions of variable size) were the most clinically relevant complications and occurred in 30 patients after 31 procedures (8.9\% per patient; $5.8 \%$ per operation), including $26 / 447$ (5.8\%) for indirect bypass, 4/70 (5.7\%) for direct bypass, and $1 / 16(6.25 \%)$ for combined bypass. Therefore, for indirect bypass and non-indirect bypass, the incidence of ischemic events was both 5.8\% (26/447 and 5/86, Table 2). In addition, CHS was observed in 4 patients after 4 procedures (1.2\% per patient; $0.8 \%$ per operation). All CHS occurred after non-indirect bypass (3 direct bypass and 1 combined bypass), and its incidence was significantly higher than that after indirect bypass ( $4.7 \mathrm{vs}$ $0.0 \%, p=0.001$ ).

The other complications were as follows: 6 subcutaneous effusions (1.1\% per operation, including 1 for direct bypass, 4 for EDAS, and 1 for MBH), 6 intracranial infections (1.1\% per operation, including 2 for direct bypass, 3 for EDAS, and 1 for $\mathrm{MBH}$ ), 5 seizures (0.9\% per operation, all 5 for EDAS), 3 instances of impaired wound healing $(0.6 \%$ per operation, including 1 for combined bypass, and 2 for EDAS), and 1 subdural hematoma ( $0.2 \%$ per operation, 1 for direct bypass) (some patients had two or more complications after one operation). No patient died due to these complications.

\section{Risk factors for postoperative ischemic events}

Among the 31 postoperative ischemic events, there were 22 TIAs (one patient had TIAs after both procedures) and 9 cerebral infarctions. Notably, all 21 patients who suffered TIAs made a full recovery by 3 months after the operation. Among the 9 patients who suffered cerebral infarction, 4 had mild disability (mRS 2), 4 had moderate disability (mRS 3), and 1 had an unfavorable outcome (mRS 4). Univariate logistic regression analyses of the preoperative clinical variables showed that older age at operation (OR 1.093, 95\% CI 0.991-1.206, $p=0.074$ ) and PCA involvement (OR 1.985, 95\% CI 0.916-4.303, $p=0.082$ ) may be associated with postoperative ischemic events (Table 3). After adjusting for all potential covariables, including sex, Suzuki stage, middle meningeal artery (MMA) collateral, occipital artery (OA) collateral, and surgical modalities, multivariate logistic regression analyses revealed that older age at operation (OR 1.129, 95\% CI 1.011-1.260, $p=0.032$ ) and PCA involvement (OR $2.587,95 \%$ CI $1.030-6.496, p=0.043$ ) were predictors of postoperative ischemic complications. 
Table 1 Baseline characteristics of hemispheres with and without postoperative complications

\begin{tabular}{|c|c|c|c|c|}
\hline \multirow[t]{2}{*}{ Characteristics } & \multirow[t]{2}{*}{ Total $(n=533)$} & \multicolumn{2}{|c|}{ Postop Complications } & \multirow[t]{2}{*}{$P$ value } \\
\hline & & Present $(n=51)$ & Absent $(n=482)$ & \\
\hline Mean age at op (yrs) & $9.6 \pm 3.7$ & $10.9 \pm 4.1$ & $9.5 \pm 3.6$ & 0.008 \\
\hline Sex, female/male ratio & $272 / 261$ & $27 / 24$ & $245 / 237$ & 0.774 \\
\hline \multicolumn{5}{|l|}{ Clinical presentation } \\
\hline Ischemic & $366(68.7)$ & $31(60.8)$ & $335(69.5)$ & 0.429 \\
\hline Infarction & $132(24.8)$ & $12(23.5)$ & $120(24.9)$ & \\
\hline $\mathrm{TIA}$ & $234(43.9)$ & $19(37.3)$ & $215(44.6)$ & \\
\hline Hemorrhagic & $28(5.3)$ & $3(5.9)$ & $25(5.2)$ & \\
\hline Nonspecific & $139(26.1)$ & $17(33.3)$ & $122(25.3)$ & \\
\hline Headache & $38(7.1)$ & $6(11.8)$ & $32(6.6)$ & \\
\hline Epilepsy & $38(7.1)$ & $5(9.8)$ & $33(6.8)$ & \\
\hline Asymptomatic & $63(11.8)$ & $6(11.8)$ & $57(11.8)$ & \\
\hline \multicolumn{5}{|l|}{ Admission mRS score } \\
\hline Mean & $1.4 \pm 0.6$ & $1.5 \pm 0.6$ & $1.4 \pm 0.6$ & 0.260 \\
\hline $0-1$ & 371 (69.6) & $31(60.8)$ & $340(70.5)$ & 0.150 \\
\hline $2-5$ & $162(30.4)$ & $20(39.2)$ & $142(29.5)$ & \\
\hline \multicolumn{5}{|l|}{ Past medical history } \\
\hline Family history & $38(7.1)$ & $4(7.8)$ & $34(7.1)$ & 1.000 \\
\hline Hypertension & $7(1.3)$ & $1(2.0)$ & $6(1.2)$ & 1.000 \\
\hline Thyroid disease & $4(0.8)$ & $1(2.0)$ & $3(0.6)$ & 0.841 \\
\hline Hyperlipidemia & $3(0.6)$ & $0(0.0)$ & $3(0.6)$ & 1.000 \\
\hline Smoking \& Alcohol use & $2(0.4)$ & $0(0.0)$ & $2(0.4)$ & 1.000 \\
\hline Diabetes & $1(0.2)$ & $0(0.0)$ & $1(0.2)$ & 1.000 \\
\hline \multicolumn{5}{|l|}{ Suzuki stage ${ }^{a}$} \\
\hline Mean & $2.9 \pm 0.9$ & $3.0 \pm 0.8$ & $2.9 \pm 0.9$ & 0.238 \\
\hline 1 & $12(2.4)$ & $0(0.0)$ & $12(2.7)$ & 0.268 \\
\hline 2 & $154(31.1)$ & $14(29.2)$ & $140(31.3)$ & \\
\hline 3 & $216(43.6)$ & $20(41.7)$ & $196(43.8)$ & \\
\hline 4 & $96(19.4)$ & $12(25.0)$ & $84(18.8)$ & \\
\hline 5 & $17(3.4)$ & $2(4.2)$ & $15(3.4)$ & \\
\hline PCA involvement ${ }^{\mathrm{a}}$ & $116(23.4)$ & $18(37.5)$ & $98(21.9)$ & 0.015 \\
\hline \multicolumn{5}{|l|}{ Collateral circulation ${ }^{\mathrm{a}}$} \\
\hline Mean & $7.0 \pm 1.8$ & $6.3 \pm 2.1$ & $7.0 \pm 1.8$ & 0.019 \\
\hline Grade I (0-4) & $54(10.9)$ & $12(25.0)$ & $42(9.4)$ & \\
\hline Grade II (5-8) & $342(69.1)$ & $26(54.2)$ & $316(70.7)$ & \\
\hline Grade III (9-12) & $99(20.0)$ & $10(20.8)$ & $89(19.9)$ & \\
\hline \multicolumn{5}{|l|}{ ECA collateral $^{a}$} \\
\hline STA collateral & $4(0.8)$ & $1(2.1)$ & $3(0.7)$ & 0.849 \\
\hline MMA collateral & $197(39.8)$ & $21(43.8)$ & $176(39.4)$ & 0.556 \\
\hline OA collateral & $29(5.9)$ & $4(8.3)$ & $25(5.6)$ & 0.656 \\
\hline \multicolumn{5}{|l|}{ Surgical modalities } \\
\hline Indirect bypass & $447(83.9)$ & $40(78.4)$ & $407(84.4)$ & 0.540 \\
\hline Direct bypass & $70(13.1)$ & $9(17.6)$ & $61(12.7)$ & \\
\hline Combined bypass & $16(3.0)$ & $2(3.9)$ & $14(2.9)$ & \\
\hline Mean LOS (days) & $15(13-18)$ & $20.0 \pm 8.8$ & $15.3 \pm 3.8$ & 0.000 \\
\hline \multicolumn{5}{|l|}{ Discharge mRS score } \\
\hline Mean & $1.4 \pm 0.6$ & $1.7 \pm 0.8$ & $1.3 \pm 0.6$ & 0.000 \\
\hline $0-1$ & $378(70.9)$ & $22(43.1)$ & $356(73.9)$ & 0.000 \\
\hline $2-5$ & $155(29.1)$ & $29(56.9)$ & $126(26.1)$ & \\
\hline
\end{tabular}

ECA External carotid artery, LOS Length of hospital stay, MMA Middle meningeal artery, OA Occipital artery, PCA Posterior cerebral artery, STA Superficial temporal artery, TIA Transient ischemic attacking

a 495 hemispheres received DSA 
Table 2 Postoperative complications stratified by surgery modalities

\begin{tabular}{|c|c|c|c|c|}
\hline \multirow[t]{2}{*}{ Complications } & \multirow[t]{2}{*}{ Total $(n=533)$} & \multicolumn{2}{|c|}{ Surgical modalities } & \multirow[t]{2}{*}{$P$ value } \\
\hline & & IB $(n=447)$ & $\mathrm{DB}$ and $\mathrm{CB}(n=86)$ & \\
\hline Total & $51(9.6)$ & $40(8.9)$ & $11(12.8)$ & 0.267 \\
\hline Ischemic events & $31(5.8)$ & $26(5.8)$ & $5(5.8)$ & 0.999 \\
\hline Infarction & $9(1.7)$ & $6(1.3)$ & $3(3.5)$ & 0.338 \\
\hline $\mathrm{TIA}$ & $22(4.1)$ & $20(4.5)$ & $2(2.3)$ & 0.534 \\
\hline Cerebral hyperfusion syndrome & $4(0.8)$ & $0(0.0)$ & $4(4.7)$ & 0.001 \\
\hline Subcutaneous effusion & $6(1.1)$ & $5(1.1)$ & $1(1.2)$ & 1.000 \\
\hline Intracranial infection & $6(1.1)$ & $4(0.9)$ & $2(2.3)$ & 1.000 \\
\hline Seizure & $5(0.9)$ & $5(1.1)$ & $0(0.0)$ & 1.000 \\
\hline Impaired wound healing & $3(0.6)$ & $2(0.4)$ & $1(1.2)$ & 0980 \\
\hline Subdural hematoma & $1(0.2)$ & $0(0.0)$ & $1(1.2)$ & 0.161 \\
\hline
\end{tabular}

$I B$ Indirect bypass, $D B$ Direct bypass, $C B$ Combined bypass

To assess the probability of stroke after revascularization surgery in pediatric MMD patients, a nomogram was constructed based on the risk factors identified by the multivariable analysis (Fig. 1).

\section{Discussion}

Our study showed that the incidence of the postoperative complication was $9.6 \%(51 / 533)$ in pediatric MMD patients. The most common complication was ischemic events $(5.8 \%, 31 / 533)$. Older age at operation and PCA involvement were identified as predictors of postoperative ischemic events. Moreover, postoperative complications were significantly related to longer hospital stays and worse outcome at discharge.

Surgical revascularization is an effective treatment for MMD in preventing recurrent stroke and to improving prognosis, especially in the pediatric population [4, 14]. Previous studies have demonstrated that indirect, direct, and combined bypass techniques are all effective options for revascularization in pediatric MMD, but each procedure has its own advantages and disadvantages [5, 14-16]. For example, according to a meta-analysis, the estimated stroke rate was higher in indirect bypass (9.0\%) than in direct bypass (4.5\%) and combined bypass (6.0\%) [14]. Our recent study showed that during the short term after surgery, direct bypass might be superior to indirect bypass in preventing recurrent ischemic strokes [15]. In addition, it usually takes months to improve cerebral blood flow after indirect bypass because neovascularization from connective tissue is not immediate [4]. However, direct and combined bypass are technically difficult, requiring more training and experience, especially in pediatric patients, due to the small caliber of the donor and recipient arteries [17]. Moreover, it has been reported that postoperative $\mathrm{CHS}$ often develops after combined and direct bypass surgery $[13,18,19]$. In the current study, all instances of CHS occurred in direct and combined bypass, and the incidence was significantly higher than that in indirect bypass.

Postoperative ischemic events may lead to potential neurological deterioration or permanent neurological deficits $[8,14,20]$. Ha et al. reported that the incidence of postoperative infarctions was $12 \%$ per patient and $6 \%$ per operation after 1283 operations; surgery-related infarctions were the most common complications and were closely related to an unfavorable outcome [7]. Kim et al. reported that that the incidence of the postoperative infarctions was $13 \%$ per patient and $6 \%$ per operation after 845 operations [5]. Bao et al. reported that the incidence of postoperative TIA or infarctions was $4.8 \%$ per patient and $2.7 \%$ per operation after 512 operations [6]. In our study, the incidence of postoperative ischemic events was $8.9 \%$ per patient and $5.8 \%$ per operation after 533 procedures, which was consistent with previous reports.

Regarding postoperative complications in pediatric MMD, there have only been a few reports with a small series of patients enrolled, and the evidence on risk factors is limited [12, 21, 22]. Muraoka et al. enrolled 58 revascularizations in 37 children and found that preoperative cerebral infarctions, younger age, higher Suzuki grade, and PCA involvement were associated with postoperative complications [21]. In this study, we also found that age at operation and PCA involvement were predictors of postoperative ischemic events. The difference is that we found that older age at operation is a risk factor for postoperative ischemic events, and we speculate that this difference might be attributed to different populations and different numbers of cases. Moreover, the leptomeningeal system from the PCA 
Table 3 Logistic regression analysis for postoperative ischemic events

\begin{tabular}{|c|c|c|c|c|c|}
\hline \multirow[t]{2}{*}{ Characteristics } & \multicolumn{2}{|c|}{ Postop Ischemic Events } & \multicolumn{2}{|c|}{$P$ value } & \multirow[t]{2}{*}{ OR $(95 \% \mathrm{Cl})$} \\
\hline & Present $(n=31)$ & Absent $(n=502)$ & Uni & Multi & \\
\hline Mean age at op (yrs) & $10.8 \pm 4.0$ & $9.5 \pm 3.7$ & 0.074 & 0.032 & $1.129(1.011-1.260)$ \\
\hline Sex, female/male ratio & $17 / 14$ & $255 / 247$ & 0.662 & 0.679 & $1.174(0.550-2.504)$ \\
\hline Ischemic presentation & $19(61.3)$ & $347(69.1)$ & 0.707 & & \\
\hline \multicolumn{6}{|l|}{ Admission mRS score } \\
\hline Mean & $1.4 \pm 0.6$ & $1.4 \pm 0.6$ & 0.789 & & \\
\hline $0-1$ & $20(64.5)$ & $351(30.1)$ & & & \\
\hline $2-5$ & $11(35.5)$ & $151(69.9)$ & & & \\
\hline \multicolumn{6}{|l|}{ Past medical history } \\
\hline Family history & $2(6.5)$ & $36(7.2)$ & 0.880 & & \\
\hline Hypertension & $1(3.2)$ & $6(1.2)$ & 0.355 & & \\
\hline Thyroid disease & $1(3.2)$ & $3(0.6)$ & 0.143 & & \\
\hline Hyperlipidemia & $0(0.0)$ & $3(0.6)$ & 0.999 & & \\
\hline Smoking \& Alcohol use & $0(0.0)$ & $2(0.4)$ & 0.999 & & \\
\hline Diabetes & $0(0.0)$ & $1(0.2)$ & 1.000 & & \\
\hline \multicolumn{6}{|l|}{ Suzuki stage ${ }^{a}$} \\
\hline Mean & $3.0 \pm 0.9$ & $2.9 \pm 0.9$ & 0.522 & 0.796 & $1.061(0.677-1.662)$ \\
\hline 1 & $0(0.0)$ & $12(2.6)$ & & & \\
\hline 2 & $10(33.3)$ & $144(31.0)$ & & & \\
\hline 3 & $11(36.7)$ & $205(44.1)$ & & & \\
\hline 4 & $8(26.7)$ & $88(18.9)$ & & & \\
\hline 5 & $1(3.3)$ & $16(3.4)$ & & & \\
\hline PCA involvement ${ }^{\mathrm{a}}$ & $11(36.7)$ & $105(22.6)$ & 0.082 & 0.043 & $2.587(1.030-6.496)$ \\
\hline \multicolumn{6}{|l|}{ Collateral circulation ${ }^{a}$} \\
\hline Mean & $6.6 \pm 2.1$ & $7.0 \pm 1.8$ & 0.276 & & \\
\hline Grade I (0-4) & $6(20.0)$ & $48(10.3)$ & & & \\
\hline Grade II (5-8) & $16(53.3)$ & $326(70.1)$ & & & \\
\hline Grade III (9-12) & $8(26.7)$ & 91 (19.6) & & & \\
\hline \multicolumn{6}{|l|}{ ECA collateral $^{\mathrm{a}}$} \\
\hline STA collateral & $0(0.0)$ & $4(0.9)$ & 0.999 & & \\
\hline MMA collateral & $11(36.7)$ & $186(40.0)$ & 0.718 & 0.154 & $0.533(0.224-1.267)$ \\
\hline OA collateral & $3(10.0)$ & $26(5.6)$ & 0.326 & 0.659 & $1.365(0.343-5.433)$ \\
\hline \multicolumn{6}{|l|}{ Surgical modalities } \\
\hline Indirect bypass & $26(83.9)$ & $421(83.9)$ & 0.942 & 0.739 & $1.435(0.171-12.013)$ \\
\hline Direct bypass & $4(12.9)$ & $66(13.1)$ & 0.934 & 0.985 & 0.979 (0.099-9.718) \\
\hline Combined bypass & $1(3.2)$ & $15(3.0)$ & & & \\
\hline
\end{tabular}

ECA External carotid artery, MMA Middle meningeal artery, OA Occipital artery, PCA Posterior cerebral artery, STA Superficial temporal artery

a 495 hemispheres received DSA

plays an important role against ischemic and hemorrhagic stroke in MMD [10, 23]. PCA involvement may suggest failed compensatory collateralization. When undergoing a surgical revascularization, patients with PCA involvement may be more vulnerable than patients without PCA involvement, and have a higher incidence of postoperative ischemic events. Therefore, patients with older age at operation or PCA involvement need special attention in perioperative management.
Our previous study reported that advanced Suzuki stage and preoperative ischemic presentation weres associated with postoperative ischemic complications in adult MMD patients [8]. However, the present study showed that Suzuki stage and ischemic presentation were not risk factors in the pediatric population. One possible explanation is that most pediatric patients receive indirect bypass, but many adult patients in our institute receive direct and combined bypass. For patients with an advanced Suzuki 


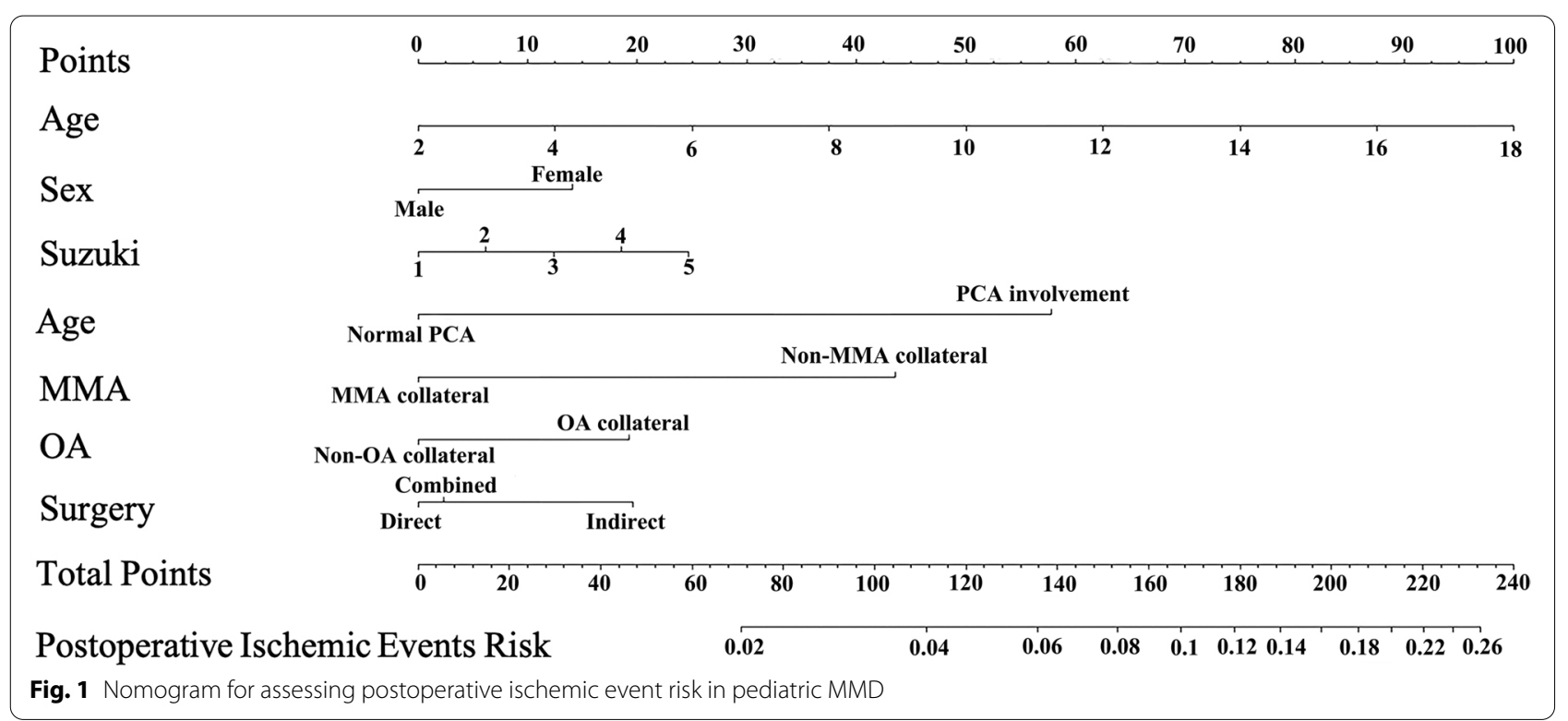

stage, direct and combined bypass might be difficult due to the small caliber of the recipient artery [4], which may increase the risk of complications. Moreover, the majority of pediatric patients presented with ischemia, and the proportion of hemorrhagic-type patients was significantly lower in the pediatric population $(5.3 \%$ in the current study) than in the adult population (32.1\% in our previous study) [24]. This may explain why ischemia at presentation was not associated with postoperative ischemic events in pediatric MMD patients.

There have recently been some studies on perioperative management to prevent postoperative ischemic events in pediatric MMD patients. For example, Honjo et al. tried to use dexmedetomidine to prevent crying after surgical revascularization in pediatric patients [25]. The study of Lee et al.showed that dysfunctional intraoperative BP autoregulation may increase the risk of TIA in pediatric patients $[12,26]$. Perioperative care with BP control combined with the administration of aspirin may reduce the potential risk of postoperative ischemic complications [16]. These studies are interesting and might be useful to prevent postoperative ischemic events in pediatric patients.

The present study has some limitations. First, this study was retrospectively carried out in a single center, and although the number of cases was large, selection bias may exist. Second, not all patients underwent DSA examination, and we could not evaluate preoperative collateral circulation in all patients. Third, the number of patients who received direct and combined bypass was small. Fourth, RNF213 was not sequenced, and the effect of the variants on postoperative ischemic events remains unknown. Further prospective studies are still needed to determine the predictors of postoperative ischemic complications in pediatric MMD patients.

\section{Conclusion}

Older age at operation and posterior cerebral artery involvement are risk factors for postoperative ischemic events in pediatric MMD patients.

\section{Abbreviations}

ACA: Anterior cerebral artery; CHS: Cerebral hyperperfusion syndrome: DSA: Digital subtraction angiography; ECA: External carotid artery; EDAS: Encephaloduroarteriosynangiosis; LOS: Length of hospital stays; MBH: Multiple burr hole; MCA: Middle cerebral artery; MMA: Middle meningeal artery; MMD: Moyamoya disease; mRS: Modified rankin scale; PCA: Posterior cerebral artery; STA: Superficial temporal artery; TIA: Transient ischemic attack; VA: Vertebrobasilar artery

\section{Acknowledgements}

We thank Drs. Chang Liu, Yonggang Ma, Cunxin Tan, Pengjun Jiang, Rutao Luo, Gongyi Yu, Zhiyong Shi, Lebao Yu, and Junshi Shao for their contributions to the data collection.

\section{Authors' contributions}

$X D, Y Z$, and JZ: conception and design. PG and XD: acquisition of data. PG and $X D$ : analysis and interpretation of data. $X D, Y Z$, and $P G$ : drafting the article. $R W, Y Z$, and DZ: technical supports and surgery. All authors critically revising the article and approved the final version of the manuscript. YZ and JZ: study supervision.

\section{Funding}

This study was supported by National Natural Science Foundation of China (81870833). The funders had no role in study design, data collection and analysis, interpretation of data and in writing the manuscript.

\section{Availability of data and materials}

The datasets supporting the conclusions of this study are available from the corresponding author on reasonable request. 


\section{Declarations}

\section{Ethics approval and consent to participate}

The study was approved by Beijing Tiantan Hospital Ethics Committee, Capital medical university (Ethics review number: KY2016-048-01). We received appropriate approval and consent to utilize the raw data for this study from Beijing Tiantan Hospital, Capital Medical University.

\section{Consent for publication}

Not applicable.

\section{Competing interests}

The authors declare that they have no competing interests.

\section{Author details}

'Department of Neurosurgery, Beijing Tiantan Hospital, Capital Medical University, Beijing 100070, China. ${ }^{2}$ China National Clinical Research Center for Neurological Diseases, Beijing, China. ${ }^{3}$ Center of Stroke, Beijing Institute for Brain Disorders, Beijing, China. ${ }^{4}$ Beijing Key Laboratory of Translational Medicine for Cerebrovascular Disease, Beijing, China. ${ }^{5}$ Savaid Medical School, University of Chinese Academy of Sciences, Beijing, China.

Received: 4 December 2020 Accepted: 10 June 2021

Published online: 22 June 2021

\section{References}

1. Scott RM, Smith ER. Moyamoya disease and moyamoya syndrome. N Engl J Med. 2009;360(12):1226-37.

2. Suzuki J, Takaku A. Cerebrovascular "moyamoya" disease. Disease showing abnormal net-like vessels in base of brain. Arch Neurol. 1969;20(3):288-99.

3. Kim JS. Moyamoya disease: epidemiology, clinical features, and diagnosis. J Stroke. 2016:18(1):2-11.

4. Kim T, Oh CW, Bang JS, Kim JE, Cho WS. Moyamoya disease: treatment and outcomes. J Stroke. 2016;18(1):21-30.

5. Kim SK, Cho BK, Phi JH, Lee JY, Chae JH, Kim KJ, Hwang YS, Kim IO, Lee DS, Lee J, Wang KC. Pediatric moyamoya disease: an analysis of 410 consecutive cases. Ann Neurol. 2010;68(1):92-101.

6. Bao XY, Duan L, Yang WZ, Li DS, Sun WJ, Zhang ZS, Zong R, Han C. Clinical features, surgical treatment, and long-term outcome in pediatric patients with moyamoya disease in China. Cerebrovasc Dis. 2015;39(2):75-81.

7. Ha EJ, Kim KH, Wang KC, Phi JH, Lee JY, Choi JW, Cho BK, Yang J, Byun YH, Kim SK. Long-Term outcomes of indirect bypass for 629 children with moyamoya disease: longitudinal and cross-sectional analysis. Stroke. 2019;50(11):3177-83.

8. Zhao M, Deng X, Zhang D, Wang S, Zhang Y, Wang R, Zhao J. Risk factors for and outcomes of postoperative complications in adult patients with moyamoya disease. J Neurosurg. 2019:130:531-52.

9. Research Committee on the Pathology and Treatment of Spontaneous Occlusion of the Circle of Willis; Health Labour Sciences Research Grant for Research on Measures for Infractable Diseases. Guidelines for diagnosis and treatment of moyamoya disease (spontaneous occlusion of the circle of Willis). Neurol Med Chir (Tokyo). 2012;52(5):245-66.

10. Liu ZW, Han C, Zhao F, Qiao PG, Wang H, Bao XY, Zhang ZS, Yang WZ, Li DS, Duan L. Collateral circulation in moyamoya disease: a new grading system. Stroke. 2019;50(10):2708-15

11. Deng X, Gao F, Zhang D, Zhang Y, Wang R, Wang S, Cao Y, Zhao Y, Pan Y, Ye X, Liu X, Zhang Q, Wang J, Yang Z, Zhao M, Zhao J. Effects of different surgical modalities on the clinical outcome of patients with moyamoya disease: a prospective cohort study. J Neurosurg. 2018;128(5):1327-37.
12. Fujimura M, Inoue T, Shimizu H, Saito A, Mugikura S, Tominaga T. Efficacy of prophylactic blood pressure lowering according to a standardized postoperative management protocol to prevent symptomatic cerebral hyperperfusion after direct revascularization surgery for moyamoya disease. Cerebrovasc Dis. 2012:33(5):436-45.

13. Zhao WG, Luo Q, Jia JB, Yu JL. Cerebral hyperperfusion syndrome after revascularization surgery in patients with moyamoya disease. Br J Neurosurg. 2013;27(3):321-5.

14. Ravindran K, Wellons JC, Dewan MC. Surgical outcomes for pediatric moyamoya: a systematic review and meta-analysis. J Neurosurg Pediatr. 2019:1-10.

15. Zhao Y, Lu J, Yu S, Li J, Deng X, Zhang Y, Zhang D, Wang R, Wang H, Zhao $Y$. Comparison of long-term effect between direct and indirect bypass for pediatric ischemic-type moyamoya disease: a propensity score-matched study. Front Neurol. 2019;10:795.

16. Rashad S, Fujimura M, Niizuma K, Endo H, Tominaga T. Long-term followup of pediatric moyamoya disease treated by combined direct-indirect revascularization surgery: single institute experience with surgical and perioperative management. Neurosurg Rev. 2016;39(4):615-23.

17. Kim H, Jang DK, Han YM, Sung JH, Park IS, Lee KS, Yang JH, Huh PW, Park YS, Kim DS, Han KD. Direct bypass versus indirect bypass in adult moyamoya angiopathy with symptoms or hemodynamic instability: a meta-analysis of comparative studies. World Neurosurg. 2016;94:273-84.

18. Fujimura M, Shimizu H, Inoue T, Mugikura S, Saito A, Tominaga T. Significance of focal cerebral hyperperfusion as a cause of transient neurologic deterioration after extracranial-intracranial bypass for moyamoya disease: comparative study with non-moyamoya patients using $\mathrm{N}$-isopropyl$\mathrm{p}$-[(123)l]iodoamphetamine single-photon emission computed tomography. Neurosurgery. 2011;68(4):957-64. discussion 964-965.

19. Hwang JW, Yang HM, Lee H, Lee HK, Jeon YT, Kim JE, Lim YJ, Park HP. Predictive factors of symptomatic cerebral hyperperfusion after superficial temporal artery-middle cerebral artery anastomosis in adult patients with moyamoya disease. Br J Anaesth. 2013;110(5):773-9.

20. Kim SH, Choi JU, Yang KH, Kim TG, Kim DS. Risk factors for postoperative ischemic complications in patients with moyamoya disease. J Neurosurg. 2005;103(5 Suppl):433-8

21. Muraoka S, Araki Y, Kondo G, Kurimoto M, Shiba Y, Uda K, Ota S, Okamoto S, Wakabayashi T. Postoperative cerebral infarction risk factors and postoperative management of pediatric patients with moyamoya disease. World Neurosurg. 2018;113:e190-190e199.

22. Kim HG, Lee SK, Lee JD. Characteristics of infarction after encephaloduroarteriosynangiosis in young patients with moyamoya disease. Neurosurg Pediatr. 2017;19(1):1-7.

23. Baltsavias $\mathrm{G}$, Khan N, Valavanis A. The collateral circulation in pediatric moyamoya disease. Childs Nerv Syst. 2015;31(3):389-98.

24. Ge P, Zhang Q, Ye X, Wang S, Zhang D, Zhao J. Clinical features, surgical treatment, and long-term outcome in children with hemorrhagic moyamoya disease. J Stroke Cerebrovasc Dis. 2018;27(6):1517-23.

25. Honjo K, Osato T, Omori S, Muraki T, Ishikawa K, Okamura N, Nakamura $H$. Preventing crying after revascularization surgery in pediatric patients with moyamoya disease: sedation with dexmedetomidine. No Shinkei Geka. 2019;47(5):525-30.

26. Lee JK, Williams M, Reyes M, Ahn ES. Cerebrovascular blood pressure autoregulation monitoring and postoperative transient ischemic attack in pediatric moyamoya vasculopathy. Paediatr Anaesth. 2018;28(2):94-102.

\section{Publisher's Note}

Springer Nature remains neutral with regard to jurisdictional claims in published maps and institutional affiliations. 